\title{
AFFINE LAMINATIONS AND THEIR STRETCH FACTORS
}

\author{
ULRICH OERTEL
}

\begin{abstract}
A lamination $L$ embedded in a manifold $M$ is an affine lamination if its lift $L$ to the universal cover $\tilde{M}$ of $M$ is a measured lamination and each covering translation multiplies the measure by a factor given by a homomorphism, called the stretch homomorphism, from $\pi_{1}(M)$ to the positive real numbers. There is a method for analyzing precisely the set of affine laminations carried by a given branched manifold $B$ embedded in $M$. The notion of the "stretch factor" of an affine lamination is a generalization of the notion of the stretch factor of a pseudo-Anosov map. The same method that serves to analyze the affine laminations carried by $B$ also allows calculation of stretch factors.

Affine laminations occur commonly as essential 2-dimensional laminations in 3-manifolds. We shall describe some examples. In particular, we describe affine essential laminations which represent classes in real 2-dimensional homology with twisted coefficients.
\end{abstract}

The first version of this paper was written in 1988, at about the same time as the paper [HO1], which is a study of spaces of affine laminations in surfaces. Since then, there has been a renewed interest in affine laminations, see for example $[\mathbf{L}]$ and $[\mathbf{C a}]$. The current version includes a new section on affine 2-laminations in 3-manifolds. These arise, for example, as realizations of classes in the real homology with twisted coefficients of a 3-manifold. More general constructions show that affine essential laminations are, in some sense, abundant in 3-manifolds, see [GO] for background on essential laminations.

I thank Joseph Christy, Allen Hatcher, and Lee Mosher for comments, corrections, and discussions.

\section{Definitions and fundamentals.}

Let $M$ be a compact manifold of dimension $n$, possibly with boundary. A lamination $L$ is a codimension-1 foliated closed subset of M. Usually the leaves of $\mathrm{L}$ are required to be smooth, but the foliation is not required to be smooth. 
A transverse affine structure for the lamination $L$ embedded in $M$ is a transverse measure $\mu$ for the lift $\tilde{L}$ of $L$ to the universal cover $\tilde{M}$ of $M$ with the property that for every covering translation $\alpha, \alpha^{*}(\mu)=\phi(\alpha) \cdot \mu$ for some $\phi(\alpha) \in \mathbb{R}_{+}$, where $\alpha^{*}(\mu)$ denotes the pull-back of the measure $\mu$. Notice that $\phi$ must be a homomorphism $\phi: \pi_{1}(M) \rightarrow \mathbb{R}_{+}$, where $\mathbb{R}_{+}$denotes the group of positive numbers with multiplication. Choosing some number $\lambda>0$ as a base, $\log _{\lambda} \phi$ is an element $\psi \in \operatorname{Hom}\left(\pi_{1}(M), \mathbb{R}\right) \simeq H^{1}(M ; \mathbb{R})$.

The lamination $L$ together with the transverse affine structure represented by the measure $\mu$ on the lift $\tilde{L}$ is an affine lamination $(L, \mu)$. If $\phi$ is the associated stretch homomorphism and $\psi=\log _{\lambda}(\phi) \in H^{1}(M ; \mathbb{R})$, then the number $\lambda$ is the stretch factor at the cohomology class $\psi$ of the affine lamination $(L, \mu)$. Thus the stretch factor of $(L, \mu)$ at $\psi$ is the number $\lambda$ such that $\lambda^{\psi}=\phi$.

We shall abuse notation by sometimes using $L$ to denote the affine lamination $(L, \mu)$ as well as the topological lamination $L$.

Notice that the stretch factor is actually a function on the ray through the class $\psi$ in $H^{1}(M ; \mathbb{R})$. Since $\log _{\lambda}(\phi)=\psi, \lambda^{\psi}=\phi$, we conclude $\left\{\lambda^{1 / k}\right\}^{k \psi}=\phi$, which implies that the stretch factor at $k \psi$ of the affine lamination $L$ is $\lambda^{1 / k}$. The following proposition summarizes these important properties of the stretch factor.

Proposition 1.1. The stretch factor $\lambda$ for an affine lamination $(L, \mu)$ is a function on the ray through the cohomology class $\log (\phi) \in \operatorname{Hom}\left(\pi_{1}(M), \mathbb{R}\right)=$ $H^{1}(M ; \mathbb{R})$. The function $1 / \log (\lambda)$ is linear on the ray.

The reader may need some motivation for the definition of the stretch factor of an affine lamination $(L, \mu)$. The most accessible explanation of affine laminations and stretch factors involves branched manifolds, which will be introduced later in the paper. In the meantime, we attempt to explain the stretch factor using the formal definitions. The stretch factor is a "multiplicative growth rate" of the transverse measure $\mu$ on $\tilde{L}$. Suppose for simplicity that the cohomology class $\psi$ is an integer lattice point. In this case, choose a codimension- 1 transversely oriented submanifold $S$ to represent the class. A simple argument shows that if $\psi$ is "primitive", i.e. is not a positive integer multiple of another integer lattice class, then $S$ can be chosen to be connected. So suppose $\psi$ is primitive, cut $M$ open on $S$, then build a $\mathbb{Z}$-cover $\hat{M}$ by glueing a $\mathbb{Z}$ 's worth of copies of the cut-open manifold in a linear pattern. The transverse measure $\mu$ descends to the lift $\hat{L}$ of $L$ to this intermediate cover, and the generator, $\alpha$, say, of the group of covering translations, takes $\hat{L}$ to itself, not preserving $\mu$, but multiplying it by a constant, so that the pull-back $\alpha^{*}(\mu)=\lambda(\psi) \mu$, where $\lambda(\psi)$ is the stretch factor at $\psi$. The dependence of $\lambda$ on the choice of cohomology class 
in the ray can be illustrated by considering a non-primitive class $\zeta=r \psi$. In this case, see [T], any transversely oriented submanifold $R$ representing the class has exactly $r$ components which are not null-homologous, and we may of course choose to let $R$ be $r$ copies of $S$. Now consider the same cover as before; when we find the stretch factor at $\zeta$, the covering translation $\alpha$ is deemed to move the lamination $\hat{L}$ a "distance" $r$, as measured by the cohomology class $\zeta$, so the dilatation in the measure per unit of distance is smaller, given by the formula $\alpha^{*}(\mu)=(\lambda(\zeta))^{r} \mu=\lambda(\psi) \mu$. We conclude that if $\zeta=r \psi$, then $(\lambda(\zeta))^{r}=\lambda(\psi)$.

Historically, the motivation for these definitions comes from a special family of affine laminations, namely suspended stable laminations for pseudoAnosov maps. Suppose $M$ is a 3-manifold which fibers over $S^{1}$ with fiber $F$, and suppose the mondoromy is a pseudo-Anosov map $\rho: F \rightarrow F$. Then the suspended stable lamination $L$ (the mapping torus of the stable lamination) is an affine lamination, as we shall see later. The stretch factor of $L$ evaluated at the cohomology class represented by an oriented fiber $F$ in $M$ is the usual stretch factor of the pseudo-Anosov map $\rho$. Commonly, manifolds which fiber over $S^{1}$ fiber in many different ways, and there is a theory due to Thurston, [T], which explains how different fibers (or fibrations) are related by the norm on homology, see also [O1]. Building on Thurston's work from a dynamical systems point of view, David Fried has studied the growth rates (stretch factors) of monodromies of related fibers, see [FLP], [F1], and [F2]. Other work on the subject includes that of Joseph Christy and Lee Mosher, see $[\mathbf{C 1}],[\mathbf{C 2}],[\mathbf{M}]$. In this paper we shall study stretch factors in the general setting of affine laminations, using only very elementary methods. The methods are applicable to the case of suspended stable laminations.

We shall use certain finite data on branched manifolds to represent affine laminations, so we begin by defining branched manifolds and related notions, see [02], [O3], or see [Wi] for original versions of some definitions.

A closed branched manifold $B$ of dimension $m=n-1$ with generic branch locus is a space with smooth structure locally modelled on spaces $X$, shown in the case $m=1$ and $m=2$ in Figure 1. (A neighborhood of each point of $B$ is diffeomorphic to a neighborhood of a point in $X$.) For arbitrary $m$ the models $X$ are constructed from stacks of $m+1$ discs, with adjacent discs in the stack identified on appropriate half-discs. The examples in this paper only involve branched manifolds of codimension-1 in surfaces and 3manifolds. The branch locus $K$ of $B$ is the union of points of $B$ none of whose neighborhoods are manifolds or manifolds with boundary. The branch locus is a cell complex of dimension $m-1$, but it can also be regarded as the image of a branch manifold of dimension $m-1$ immersed in $B$. The sectors $Z_{i}$ of $B$ are the completions (in a path metric coming from a Riemannian 
metric on $B$ ) of the components of $B-K$. If $B$ is embedded in a manifold $M$ of dimension $n$, then a fibered neighborhood $N(B)$ of $B$ in $M$ is a closed regular neighborhood of $B$ foliated by interval fibers, as shown in Figure 1, with the frontier of $N(B)$ being the union of the horizontal boundary, $\partial_{h} N(B)$, and the vertical boundary $\partial_{v} N(B)$ as shown. There is a projection map $\pi: M \rightarrow M / \sim$ which maps $M$ to a quotient space in which fibers of $N(B)$ are collapsed to points of $M / \sim$. Notice that $M / \sim$ can be identified with $M$. Notice also that $\partial_{v} N(B)$ is an I-bundle over the branch manifold of $B$, and that the branch locus is $\pi\left(\partial_{v} N(B)\right)$.

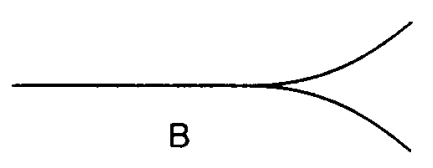

B

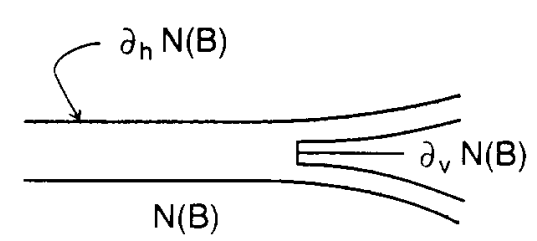

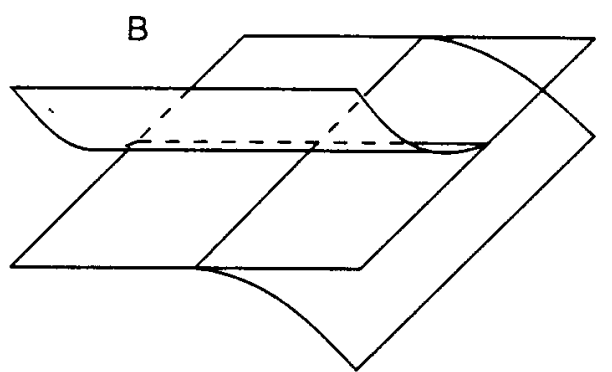

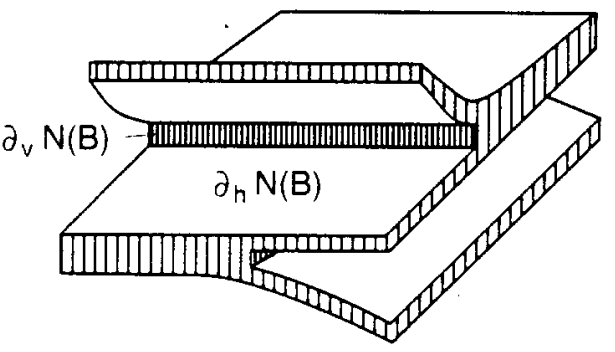

Figure 1.

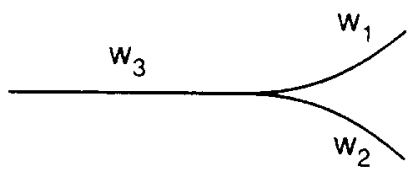

$w_{3}=w_{1}+w_{2}$

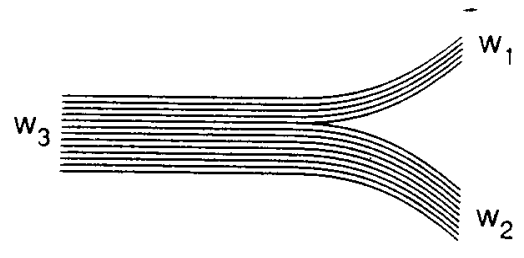

Figure 2. 
A lamination $L$ is carried by the branched manifold $B$ if $L$ can be embedded in $N(B)$ transverse to fibers. It is fully carried by $B$ if $L$ also intersects every fiber of $N(B)$. An invariant measure $w$ on $B$ is a vector which assigns a real weight $w_{i}$ to each sector $Z_{i}$ of $B$ such that the weights satisfy branch equations as shown in Figure 2. Alternatively $w$ can be regarded as a nonnegative "thickness" function $w: B \rightarrow \mathbb{R}$ which is constant on the interiors of sectors. The easiest way to describe a measured lamination corresponding to an invariant measure $w$ on a branched manifold $B$ is as a measured neighbborhood $N_{w}(B)$, as shown in Figure 2, similar to a fibered neighborhood and also having a projection map $\pi: N_{w}(B) \rightarrow B$. This is a closed singularly foliated regular neighborhood of $B$ with $\operatorname{cl}\left(\pi^{-1}\left(\stackrel{\circ}{Z}_{i}\right)\right)$ foliated in a standard way with "thickness" $w_{i}$. Leaves of $\operatorname{cl}\left(\pi^{-1}\left(\stackrel{\circ}{Z}_{i}\right)\right)$ are attached to leaves in an adjacent $\operatorname{cl}\left(\pi^{-1}\left(\stackrel{\circ}{Z}_{i}\right)\right)$ in the obvious way: Corresponding leaves have the same distance from a common boundary leaf. The foliation of $N_{w}(B)$ has cusp singularities on a manifold $\Upsilon \subset \partial N_{w}(B)$ of dimension $m-1$ homeomorphic to the branch manifold. The neighborhood $N_{w}(B)$ can be changed to an actual measured lamination by slitting open $N_{w}(B)$ on the leaves of $N_{w}(B)-\Upsilon$ abutting $\Upsilon$ from the interior of $N_{w}(B)$.

Suppose $B$ and $B^{\prime}$ are branched manifolds embedded in $M$. We say $B$ is a splitting of $B^{\prime}$, or $B^{\prime}$ is a pinching of $B$ if the following condition holds. There is an I-bundle $J$ in $M$ over an $m$-manifold, possibly with boundary, such that $N\left(B^{\prime}\right)=N(B) \cup J$ where $J \cap N(B) \subset \partial J ; \partial_{h} J \subset \partial_{h} N(B)$; and $\partial_{v} J \cap N(B) \subset \partial_{v} N(B)$ is a finite collection of components whose fibers are fibers of $\partial_{v} N(B) . \quad\left(\partial_{v} J\right.$ denotes the pre-image of the boundary of the base manifold of $J$ under projection.) All of these definitions can easily be extended to branched manifolds with boundary, which may be properly embedded in manifolds with boundary.

Clearly now we can represent an affine lamination $L$ fully carried by $B$ as an invariant measure $w$ on $\tilde{B}$ satisfying a certain condition with respect to covering translations. We require that for each covering translation $\alpha$, $\alpha^{*} w=\phi(\alpha) w$, where $\phi$ is the stretch homomorphism $\phi: \pi_{1}(M) \rightarrow \mathbb{R}_{+}$. If one wishes to analyze all possible affine laminations carried by a given branched manifold $B$ embedded in $M$, a good method is the following.

Definition 1.2. Let $\left\{S_{1}, S_{2}, \ldots, S_{k}\right\}$ be any set of transversely oriented codimension -1 submanifolds representing cohomology classes in $M$. A set of real numbers $(\sigma, w)=\left(\sigma_{1}, \sigma_{2}, \ldots, \sigma_{k} ; w_{1}, w_{2}, \ldots, w_{s}\right)$, each $\sigma_{i}>0$ assigned to one of the $S_{i}$ and each $w_{j}$ assigned to one of the components of $B-$ [(branch locus) $\left.\cup\left(\cup_{i} S_{i}\right)\right]$, is called a broken invariant measure on $B$ (for $\left.S_{1}, \ldots, S_{k}\right)$ if the $w_{j}$ 's satisfy branch equations at the branch locus and if $w_{j}$ 's on opposite sides of $S_{i}$ differ by a factor $\sigma_{i}$, the weight $w_{+}$on the plus- 
side of $S_{i}$ equalling $\sigma_{i} w_{-}$, where $w_{-}$is the weight on the minus-side of $S_{i}$. The set of broken measures on $B$ for $S_{1}, \ldots, S_{k}$ lies in $\mathbb{R}^{k+s}$, where $s$ is the number of components of $B-\left[(\right.$ branch locus $\left.) \cup\left(\cup S_{i}\right)\right]$.

Suppose now that the $S_{i}$ 's form a basis for the integer lattice points in $H^{1}(M ; \mathbb{R})$. The set of broken measures in $\mathbb{R}^{k+s}$ becomes a non-projectivized space of broken measures which we denote $\mathcal{A}(B)$. The set of non-trivial broken measures $(\sigma ; w)$ such that $w \neq 0$ is closed under multiplication of $w$ by positive scalars. Projectivizing by taking the quotient topology for the equivalence relation $(\sigma ; r w) \sim(\sigma ; w)$ we get the (projective) space of broken measures $\mathcal{B}(B)$. We shall show later that it does not depend on the choice of $S_{i}$ 's. The projectivization can be achieved concretely as the set of broken measures satisfying $\Sigma w_{j}=1$, i.e. $\mathcal{B}(B)=\mathcal{A}(B) \cap\left(\mathbb{R}^{k} \times \Delta\right)$ where $\Delta$ is the standard $(s-1)$ - simplex in $\mathbb{R}^{s}$, given by $\Sigma w_{j}=1, w_{j} \geq 0$.

Proposition 1.3. Every broken measure on $B$ represents an affine lamination. Conversely, if $L$ is an affine lamination carried by $B$, and $S_{1}, \ldots, S_{k}$ represents a basis for $H^{1}(M ; \mathbb{R})$, then there is a broken measure on $B$ for $S_{1}, \ldots, S_{k}$ representing $L$.

Proof. Given a broken measure $(\sigma ; w)$ on $B$, we lift it to $\tilde{M}$. Let $\tilde{S}_{i}$ denote the preimage in the universal cover $\tilde{M}$ of $S_{i}$. In $\tilde{M}$, we then have a measure $f$ on $\tilde{B}-\left(\cup_{i} \tilde{S}_{i}\right)$ such that the weight on $\tilde{B}$ is multiplied by a factor $\sigma_{i}$ as one crosses the preimage $\tilde{S}_{i}$ of $S_{i}$ in the positive direction. Each component of $\tilde{S}_{i}$ separates $\tilde{M}$, so we construct an invariant measure on $\tilde{B}$ as follows. For each component $\tilde{C}$ of each $\tilde{S}_{i}$, we multiply all the weights on the positive side of $\tilde{C}$ by $1 / \sigma_{i}$, then we normalize, multiplying all weights so that the weight at some fixed base point in $\tilde{B}$ equals 1 . Doing this for all components of all $\tilde{S}_{i}$ 's, one by one, yields a measure $w$ on $\tilde{B}$. Clearly $w$ has the property that for each covering translation $\alpha, \alpha^{*} w=\phi(\alpha) \cdot w$ where $\phi \in \operatorname{Hom}\left(\pi_{1}(M), \mathbb{R}_{+}\right)$ is determined by $\sigma$.

Conversely, suppose we are given an affine lamination $L$ fully carried by $B$. Then $\tilde{L}$ is a measured lamination carried by $\tilde{B}$ which determines a measure $w$ on $\tilde{B}$ with the property that $\alpha^{*} w=\phi(\alpha) \cdot w$. Choose transversely oriented manifolds $S_{1}, \ldots, S_{k}$ representing a basis for $H^{1}(M ; \mathbb{R})$. The homomorphism $\psi=\log (\phi)$ is a cohomology class which can be written $x_{1} S_{1}+x_{2} S_{2}+\ldots+x_{k} S_{k}$. For an element $\alpha$ of $\pi_{1}(M)$, therefore, $\phi(\alpha)=$ $e^{x_{1}\left\langle\alpha, S_{1}\right\rangle} e^{x_{2}\left\langle\alpha, S_{2}\right\rangle} \ldots e^{x_{k}\left\langle\alpha, S_{k}\right\rangle}$, where $\langle\cdot, \cdot\rangle$ denotes algebraic intersection. By reversing the construction above we shall obtain a broken measure on $\tilde{B}$ which descends to a broken measure on $B$. For each component $C$ of the preimage $\tilde{S}_{i}$ of $S_{i}$ we multiply the measure on the plus-side of $C$ by $\sigma_{i}=e^{x_{i}}$ obtaining a "thickness" function $v$ on $\tilde{B}-\left(\cup_{i} \tilde{S}_{i}\right)$, then we normalize so $v=1$ at some fixed point. Doing this on each $C$, one at a time, yields an 
equivariant broken measure $v$ on $\tilde{B}$ which descends to a broken measure on $B$.

In the context of Definition 1.2, the stretch homomorphism $\phi$ (and therefore the stretch factor associated to an affine lamination) is completely determined by the $\sigma^{\prime}$ s: $\phi(\gamma)$ is a product with factors $\left(\sigma_{i}\right)^{ \pm 1}$, one $\sigma_{i}^{+1}\left(\sigma_{i}^{-1}\right)$ for each positive (negative) intersection of $\gamma$ with $S_{i}$. The non-projectivized space of broken measures is defined by inequalities of the form $w_{j} \geq 0$ and $\sigma_{i}>0$, and a homogeneous linear system of equations in $w_{j}$ 's with coefficients in $\mathbb{Z}\left[\sigma_{1}, \ldots, \sigma_{k}\right]$, the ring of polynomials in the $\sigma$ 's. In fact, each coefficient in each of these linear equations is \pm 1 or $\pm \sigma_{i}^{ \pm 1}$. After eliminating negative exponents, we have coefficients which are monomials. We can easily determine necessary and sufficient conditions on the $\sigma_{i}$ 's to ensure the existence of non-trivial solutions. They are a collection of polynomial conditions in the $\sigma_{i}$ 's coming from setting determinants equal to 0 , say $f_{1}(\sigma)=f_{2}(\sigma)=\ldots=f_{r}(\sigma)=0$.

Definition 1.4. The solution variety $\mathcal{S}(B)$ associated to the branched manifold $B$ is the variety $f_{1}(\sigma)=f_{2}(\sigma)=\ldots=f_{r}(\sigma)=0$ (determined by the necessary and sufficient conditions for the existence of solutions for the system of equations defining the non-projectivized space of broken measures) intersected with the open first orthant $\left(\sigma_{i}>0, i=1, \ldots, k\right)$ of $\mathbb{R}^{k}$. The $\sigma$-space $\mathcal{R}(B)$ is the subspace of $\mathcal{S}(B)$ consisting of $\sigma$ 's realized in broken measures; $\sigma$ is in the $\sigma$-space if there exists a non-trivial $w$ (with $w_{i} \geq 0$ ) such that $(\sigma, w)$ is a broken measure.

Proposition 1.5. The orthogonal projection of $\mathcal{A}(B) \subset\left(\mathbb{R}_{+}\right)^{k} \times \mathbb{R}^{s}$ or $\mathcal{B}(B) \subset\left(\mathbb{R}_{+}\right)^{k} \times \Delta$ of broken measures on $B$ to the first orthant of $\mathbb{R}^{k}$ is the variety $\mathcal{R}(B) \subset \mathcal{S}(B)$. If $\Pi: \mathcal{A}(B) \rightarrow\left(\mathbb{R}_{+}\right)^{k}$ (resp. $\Pi: \mathcal{B}(B) \rightarrow$ $\left.\left(\mathbb{R}_{+}\right)^{k}\right)$ denotes projection, then for fixed $\sigma, \Pi^{-1}(\sigma)$ is a cone over a convex polyhedron (resp. a convex polyhedron). Both $\mathcal{A}(B)$ and $\mathcal{B}(B)$ are homotopy equivalent to the variety $\mathcal{R}(B)$.

Proof. The statements in the proposition are almost immediate. For a fixed $\sigma$ in $\mathcal{R}(B)$ we have a homogeneous system of linear equations with real coefficients describing the broken measures with that $\sigma$. These, combined with the inequalities $w_{j} \geq 0$ give a solution set which is a cone over a convex polyhedron. The existence of a homotopy (continuously parametrized by the $\sigma$-space) which collapses each inverse image $\Pi^{-1}(\sigma)$ to a point proves the statements about homotopy equivalence.

Example 1.6. We consider one of the simplest possible examples: $M$ is the torus and $B$ is the train track (branched 1-manifold) shown in Figure 3. 
Describing the broken measures on $B$ using the weights shown, only one equation must be satisfied: $w_{1}+w_{2}+\sigma_{1} w_{1}=w_{2} / \sigma_{2}$ or $\sigma_{2} w_{2}+\sigma_{2} w_{1}+\sigma_{1} \sigma_{2} w_{1}=$ $w_{2}$. In addition we require $\sigma_{i}>0$ and $w_{i} \geq 0$. This defines the space of broken measures on $B$. In this example $\mathcal{S}(B)$ is the entire first quadrant of $\mathbb{R}^{2}$. If $\sigma=\left(\sigma_{1}, \sigma_{2}\right)$ is in $\mathcal{R}(B)$, then

$$
\frac{w_{1}}{w_{2}}=\frac{1-\sigma_{2}}{\sigma_{2}+\sigma_{1} \sigma_{2}} \geq 0
$$

which implies that $\sigma_{2} \leq 1$. Thus $\mathcal{R}(B)$ is the half-infinite strip $0<\sigma_{2} \leq 1$, $0<\sigma_{1}$.

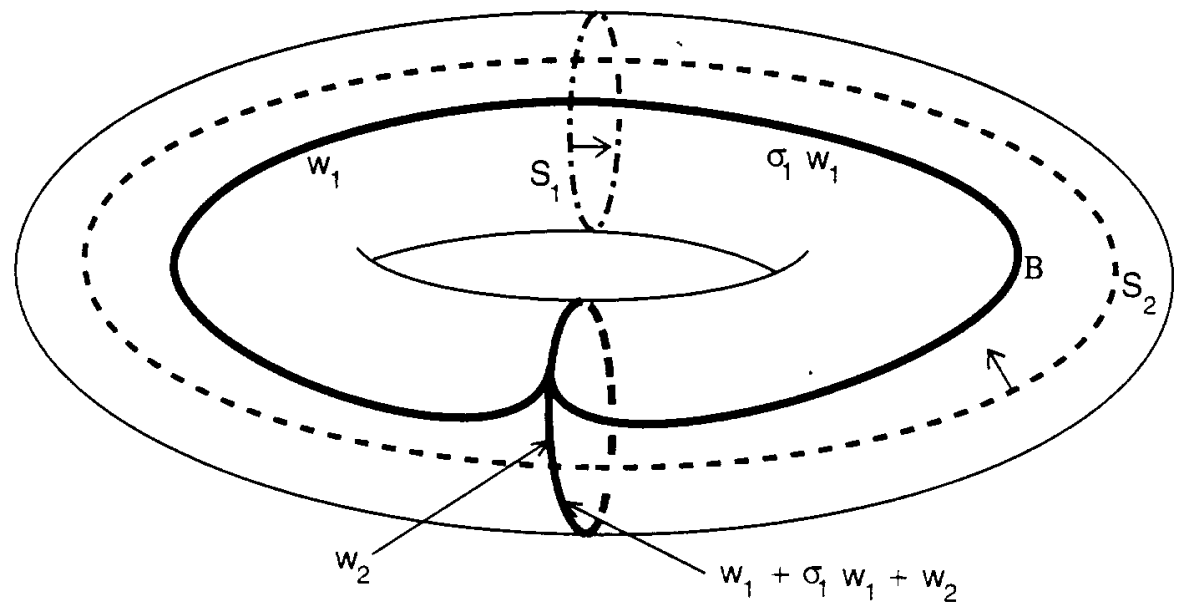

Figure 3.

If we wish to determine whether there is an affine lamination with stretch factor $\lambda$ at $\Sigma x_{i} S_{i}$ we simply substitute $\lambda^{-x_{i}}$ for $\sigma_{i}$. We have $\sigma_{2} \leq 1$, so $\lambda^{-x_{2}} \leq 1$, hence $\lambda \geq 1$ if $x_{2} \geq 0$.

Proposition 1.7. Suppose we calculate $\mathcal{A}(B)$, the non-projectivized space of broken measures, using a basis $S_{1}, \ldots, S_{k}$ over the integers for the integer lattice in $H^{1}(M ; \mathbb{R})$, and we calculate $\mathcal{A}^{\prime}(B)$ using another basis $S_{1}^{\prime}, \ldots, S_{k}^{\prime}$. Then broken measures $(\sigma, w) \in \mathcal{A}(B)$ and $\left(\sigma^{\prime}, w^{\prime}\right) \in \mathcal{A}^{\prime}(B)$ representing the same affine lamination are related by a transformation of the following type:

(1) $\sigma_{j}^{\prime}=\prod_{i=1}^{k} \sigma_{i}^{a_{i j}}$ where the matrix $\left(a_{i j}\right)$ belongs to $\mathrm{GL}(k, \mathbb{Z})$,

(2) $\quad w_{j}^{\prime}=\rho w_{j}$ where $\rho$ is a product of factors of the form $\left(\sigma_{\ell}\right)^{ \pm 1}$.

The transformation (1) is a rational map from $\left(\mathbb{R}_{+}\right)^{k}$ to $\left(\mathbb{R}_{+}\right)^{k}$ taking $\mathcal{S}(B)$ to $\mathcal{S}^{\prime}(B)$. This shows that the solution variety is independent of choices. 
The transformation (given by (1) and (2)) is a homeomorphism from $\mathcal{A}(B)$ to $\mathcal{A}^{\prime}(B)$, linear on $\sigma$-sections $\Pi^{-1}(\sigma)$, and induces a homeomorphism from $\mathcal{B}(B)$ to $\mathcal{B}^{\prime}(B)$.

Proof. Whatever our choice of basis $S_{1}, \ldots, S_{k}$, if $B$ has $s$ sectors, we choose a fixed point $p_{i}$ in each sector $Z_{i}$ where the weight corresponding to a given broken measure is $w_{i}$. Weights in all other components of $B-[$ (branch locus) $\cup$ $\left.\left(\cup S_{i}\right)\right]$ in $Z_{i}$ are then determined and have the form $\rho w_{j}$ where $\rho$ is a product of factors of the form $\left(\sigma_{\ell}\right)^{ \pm 1}$, so we omit them from our vector $w$. We represent a given affine lamination as a broken measure $(\sigma, w)$ on $B$ for $S_{1}, \ldots, S_{k}$; then we shall see what is needed to convert this broken measure to a broken measure on $B$ for $S_{1}^{\prime}, \ldots, S_{k}^{\prime}$ representing the same affine lamination. The class represented by $S_{i}^{\prime}$ can also be represented by an integer linear combination of the $S_{i}$ 's; in fact it can be represented by a manifold in a neighborhood of $\cup_{i} S_{i}$. Thus a nonsingular linear transformation $\left(a_{i j}\right)$ changing coordinates in $H^{1}(M ; \mathbb{R})$ is realized by replacing the manifolds $S_{1}, \ldots, S_{k}$ by manifolds $S_{1}^{\sharp}, \ldots, S_{k}^{\sharp}$ (each in a neighborhood of $\cup S_{i}$ ) cohomologous to manifolds $S_{1}^{\prime}, \ldots, S_{k}^{\prime}$. Since we can identify $\log \sigma_{i}$ with the coordinate $x_{i}$ of the cohomology class $x_{1} S_{1}+x_{2} S_{2}+\ldots+x_{k} S_{k}$, we can change basis in cohomology and represent our affine lamination by a broken measure on $B$ for $S_{1}^{\sharp}, \ldots, S_{k}^{\sharp}$ with the same weights $w_{i}$ and with $\sigma$-parameters $\sigma_{i}^{\prime}$ satisfying Equation (1) above. It remains now to replace the $S_{i}^{\sharp}$ 's with cohomologous $\left(S_{i}^{\prime}\right)$ 's. Since these oriented manifolds represent codimension-1 cohomology classes, each $S_{i}^{\sharp}$ can be changed to $S_{i}^{\prime}$ by a sequence of isotopies and Morse theoretic saddle events, where the saddles may have arbitrary index. There is a family $S_{i t}$ of codimension-1 manifolds which represents a sequence of isotopies interspersed with finitely many standard Morse-theoretic events with $S_{i 0}=S_{i}^{\sharp}$ and $S_{i 1}=S_{i}^{\prime}$. The intersection $S_{i t} \cap B$ can be assumed to be transverse except at finitely many isolated times when a standard saddle event occurs in the interior of a sector. For a fixed $i$, following the family $S_{i t}$ allows us to define inductively a broken measure representing our affine lamination at all times $t$. One checks that the equations defining the space of broken measures are unchanged except when $S_{i t}$ passes through a point $p_{i}$. At such a time $w_{i}$ is multiplied or divided by $\sigma_{i}^{\prime}$ (which is a product of powers of $\sigma_{\ell}$ 's), and the space of broken measures remains unchanged except for this change of one coordinate. This yields the Equation (2).

Remark. Work of Allen Hatcher and myself [HO1] shows that there is a space of all affine 1-dimensional laminations in a surface of genus $g \geq 2$. We require that the laminations be essential, i.e. that they be carried by train tracks without 0-gons, monogons, or digons. Presumably one could also define such spaces for 3-manifolds. In higher dimensions, however, it is 
not clear what an "essential" lamination should be.

Proposition 1.8. (1) Suppose the connected transversely oriented manifolds $S_{1}, S_{2}, \ldots, S_{k}$ represent a basis over $\mathbb{Z}$ of the integer lattice in $H^{1}(M, \mathbb{R})$ and let $B$ be a branched manifold in $M$. Suppose $f_{1}, \ldots, f_{r}$ are polynomials in $\sigma$ 's which define $\mathcal{S}(B)$. Then the stretch factor $\lambda$ at $x_{1} S_{1}+\cdots+x_{k} S_{k}$ of an affine lamination carried by $B$ satisfies $f_{i}\left(\lambda^{-x_{1}}, \lambda^{-x_{2}}, \ldots, \lambda^{-x_{k}}\right)=0$, $i=1, \ldots, r$.

Note: Only for certain affine laminations carried by $B$ is the stretch factor defined at $x_{1} S_{1}+\cdots+x_{k} S_{k}$; namely, only those with $\log (\phi)$ a positive multiple of $x_{1} S_{1}+\cdots+x_{k} S_{k}$.

(2) If $(1,1, \ldots, 1) \notin \mathcal{S}(B)$ or if $f_{i}(0,0, \ldots, 0) \neq 0$, for some $i=1, \ldots, r$, then the stretch factor at an integer lattice point of $H^{1}(M, \mathbb{R})$ of any affine lamination carried by $B$ is algebraic. (Note that if $(1,1, \ldots, 1) \notin \mathcal{S}(B)$, then $B$ carries no measured lamination.)

Proof. Statement (1) follows immediately from our earlier observation that $\sigma_{i}=\lambda^{-x_{i}}$. At an integer lattice point $n_{1} S_{1}+\cdots+n_{k} S_{k}$ the stretch factor must satisfy $f_{i}\left(\lambda^{-n_{1}}, \lambda^{-n_{2}}, \ldots, \lambda^{-n_{k}}\right)=0, i=1, \ldots, r$. Thus $\lambda$ must be the root of $r \geq 1$ polynomials, and $\lambda$ is algebraic unless these polynomials are all identically 0 . If they are identically 0 , they are 0 where $\lambda=1$; i.e., the $f_{i}$ 's are 0 when evaluated at $\sigma_{1}=\sigma_{2}=\ldots=\sigma_{k}=1$. Similarly, the $f_{i}$ 's are 0 when evaluated at $\lambda=0$ or $\sigma_{1}=\sigma_{2}=\ldots=\sigma_{k}=0$.

Example 1.6 had a solution variety consisting of all of the first quadrant. There were no conditions $f_{i}$, so Proposition 1.8 gives no information. The proposition does apply to the following example.

Example 1.9. $\quad$ Figure 4 shows a branched surface $B$ (branched manifold of dimension 2). We will analyze the affine laminations it carries, and their stretch factors. $B$ is drawn as an immersed branched surface in $\mathbb{R}^{3}$, but $B$ can actually be embedded in $\mathbb{R}^{3}$, see Figure 7 . We let $M$ be a regular neighborhood of $B$ embedded in $\mathbb{R}^{3}$. The cohomology $H^{1}(M ; \mathbb{R})$ has two generators $S_{1}$ and $S_{2}$ whose intersections with $B$ are shown. With the weights shown, one gets the single equation $\sigma_{1} w_{1}+\sigma_{2} w_{1}=w_{1}$ which yields $\mathcal{S}(B)$ with equation $\sigma_{1}+\sigma_{2}=1$. Clearly the broken measures on $B$ are parametrized by $\mathcal{S}(B)$, and there is at most one affine lamination carried by $B$ with $\log \phi$ on a given ray in $H^{1}(M ; \mathbb{R})$. The stretch factor at $x_{1} S_{1}+x_{2} S_{2}$ (of the unique affine lamination with $\log \phi$ on the ray through $x_{1} S_{1}+x_{2} S_{2}$ ) satisfies $\lambda^{-x_{1}}+\lambda^{-x_{2}}-1=0$. There is one stretch factor function defined throughout the first quadrant; its value at $\left(x_{1}, x_{2}\right)$ is the stretch factor there of the unique affine lamination $(L, \mu)$ carried by $B$ with the property that $\log \phi$ lies on the ray through $\left(x_{1}, x_{2}\right)$. Although the branched surface $B$ 
in this example carries a 1-parameter family of different affine laminations, these are all the same topologically.

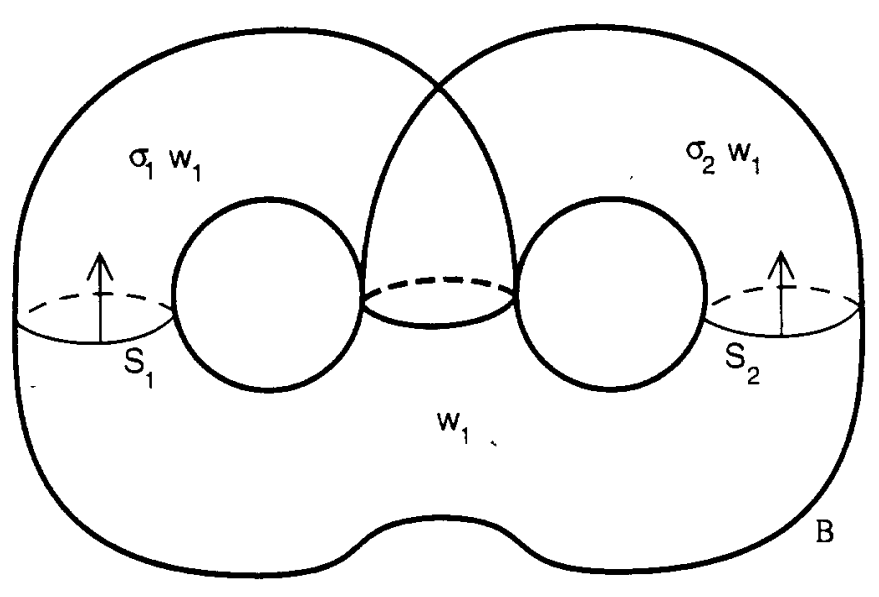

Figure 4.

\section{Mapping tori of stable laminations for pseudo-Anosov homeomorphisms.}

Branched surfaces which carry suspended stable laminations of pseudoAnosov maps are further examples to which Proposition 1.8 applies. We shall now turn to this class of examples quoting some facts about pseudoAnosov maps and their stable laminations without proof. We will also use facts about Thurston's norm on homology. The results in this section are not new, but the methods are new and much simpler than other methods used to prove the same results.

Suppose $\rho: F \rightarrow F$ is a pseudo-Anosov map and suppose $M=(F \times I)_{\rho}$, the mapping torus of $\rho$, where $\rho(F \times 1)=F \times 0$. Then the stable lamination $(\ell, \mu)$ for the pseudo-Anosov $\rho$ can also be suspended in $M$ to yield a 2dimensional lamination $L$. The lamination $L \subset M$ is constructed from $\ell \times I \subset F \times I$ by glueing $\ell \times 1$ to $\ell \times 0$ using the homeomorphism $\rho$, while $F \times 1$ is glued to $F \times 0$ using the same map $\rho$. If $\tau$ is a train track invariant for $f$ carrying $\ell$, then $(\ell, \mu)$ yields an invariant measure $v$ on $\tau$. The train track $\tau$ is mapped to $\rho(\tau)$ which can be isotoped so it is transverse to fibers in $N(\tau)$. The measure $v$ on $\tau$ pushes forward to a measure $\rho_{*} v$ on $\rho(\tau)$, and this in turn induces a measure $\lambda v$ on $\tau$, where $\lambda$ is the stretch factor 
for the pseudo-Anasov homeomorphism $\rho$. We can choose a 1-parameter family $\tau_{t}$ of train tracks, each a pinching of $\tau$, pinched more and more with increasing time, so that $\rho\left(\tau_{1}\right)=\tau=\tau_{0}$. If the family $\tau_{t}$ is sufficiently well-behaved, with $\tau_{t}$ embedded in $F \times t$, then the family yields a generic branched surface $\hat{B}$ in $F \times I$. When $\tau_{1}$ is glued to $\tau_{0}$ using $\rho$, the family yields a branched surface $B$ in $M$. Clearly $B$ fully carries the lamination $L$. The lamination $\ell \times I$ carried by $\hat{B}$ has a transverse measure, so it defines an invariant measure on $\hat{B}$. This gives a broken measure on $B$, the break occurring at the fiber $F=F \times 0=F \times 1$ with $\sigma=1 / \lambda$. We have proved that the suspended stable (or unstable) lamination $L$ for a pseudo-Anosov homeomorphism $\rho: F \rightarrow F$ is an affine lamination. The stretch factor $\lambda$ of $L$ at the class $[F]$ of the fiber is the same as the stretch factor of the pseudo-Anosov homeomorphism $\rho$. The manifold $M$ is atoroidal, so W. Thurston's norm is defined on $H_{2}(M, \partial M ; \mathbb{R}) \cong H^{1}(M ; \mathbb{R})$. The unit ball of Thurston's norm is a finite, convex polyhedron, $[\mathbf{T}]$. Thurston proved that homology classes represented by fibers are exactly the lattice points in the cones over certain open top-dimensional linear "fiber faces" of the unit sphere of the norm. Since $M$ is atoroidal and anannular, by Thurston's classification of homeomorphisms of surfaces, see [FLP], the monodromy of every fiber bundle over $S^{1}$ with total space $M$ must be pseudo-Anosov. D. Fried has shown that topologically the suspended stable lamination is the same for all fibers representing classes in the cone over a fiber face. We shall see that they are distinct as affine laminations.

The following is a corollary of Proposition 1.8, and was first proved by D. Fried.

Corollary 2.1 (D. Fried, [F1]). Suppose $M$ is a manifold which fibers over $S^{1}$, and suppose the connected transversely oriented manifolds $S_{1}, S_{2}, \ldots, S_{k}$ represent a basis over $\mathbb{Z}$ of the integer lattice in $H^{1}(M, \mathbb{R})$. Then corresponding to a given fiber face there is a polynomial $f \in \mathbb{Z}\left[\sigma_{1}, \sigma_{2}, \ldots, \sigma_{k}\right]$ (which depends on the basis) such that the stretch factor $\lambda$ of the suspended stable lamination at the class $\left[x_{1} S_{1}+\ldots+x_{k} S_{k}\right]$ of a fiber satisfies $f\left(\lambda^{-x_{1}}, \lambda^{-x_{2}}, \ldots\right.$, $\left.\lambda^{-x_{k}}\right)=0$.

The proof will follow Example 2.2. One only needs to show that one polynomial equation suffices to define the solution variety.

When choosing an example to illustrate the above corollary, it is important to use the simplest one. The one which follows was suggested to me by Joseph Christy, who has extensively studied branched surfaces which admit 
semiflows $([\mathrm{C} 1],[\mathrm{C} 2])$.

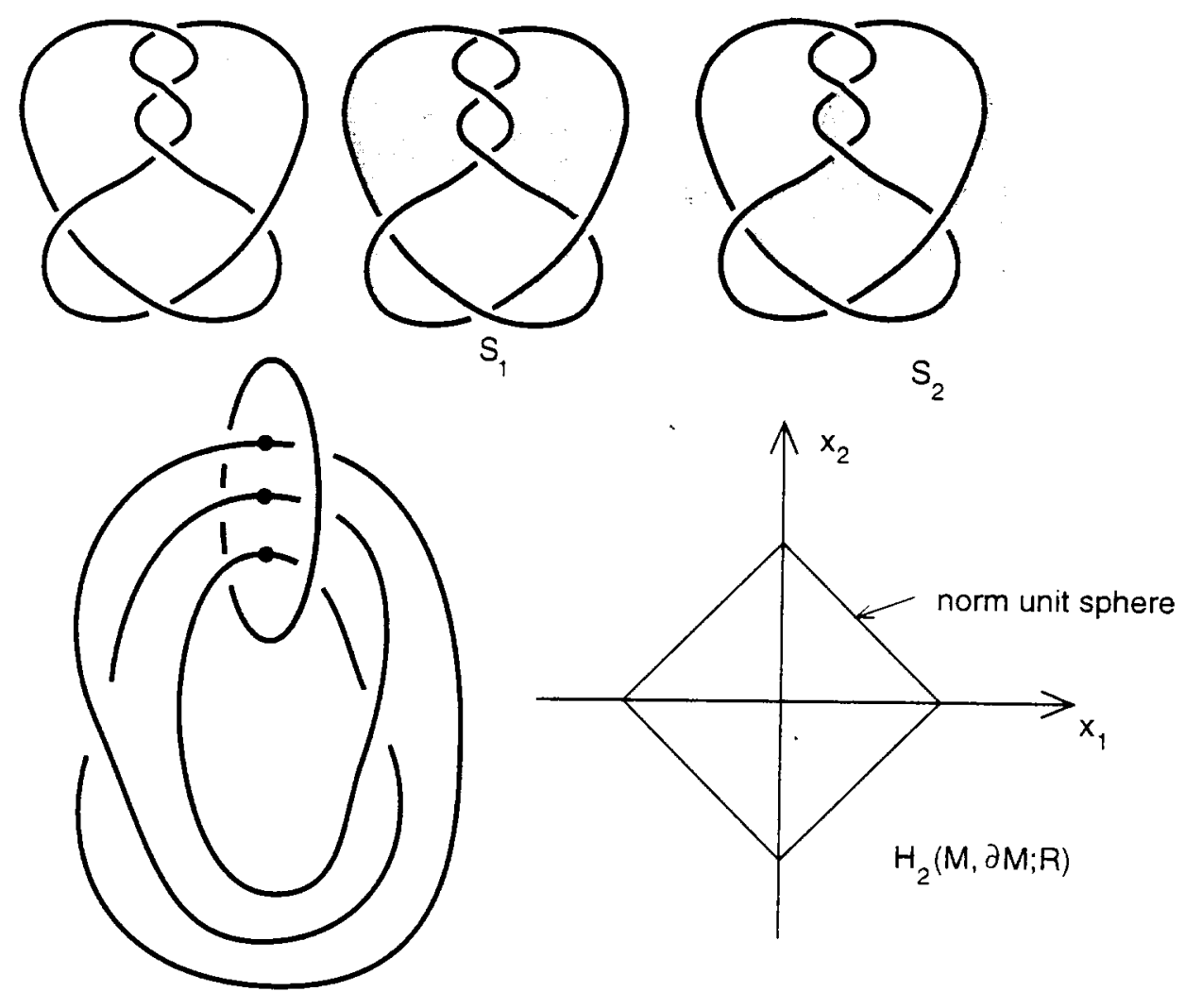

Figure 5.

Example 2.2. We let $M$ be the exterior of the link $K$ shown in Figure 5. Also shown are two orientable surfaces $S_{1}$ and $S_{2}$. The surfaces $\pm S_{1}$ and $\pm S_{2}$ are the four vertices of the unit sphere for the Thurston norm. If $S_{1}$ and $S_{2}$ are taken as a basis for $H_{2}(M, \partial M ; \mathbb{R})$ then the unit sphere is a diamond as shown. Another projection of the link (Figure 5) shows that one component of the link bounds a disc which intersects the other component in three points. Thus the link exterior fibers over the circle with fiber $F$ a 3punctured disc. Let $B$ be a branched surface fully carrying the suspended stable lamination $L$. We will find the space of broken measures for this branched surface. For this, we need to describe the intersection of $B$ with $S_{1}$ and $S_{2}$. This is most easily done by describing $B$ as a 1-parameter family of 
train tracks $\tau_{t}$ each embedded in a fiber $F \times t$, where $M=(F \times I)_{\rho}$ describes the bundle with fiber $F=$ (3-punctured disc), see Figure 5. Similarly $S_{1}$ and $S_{2}$ are described Morse theoretically in $M=(F \times I)_{\rho}$ with saddles indicated by parallel pairs of arcs. If $F$ has a transverse orientation towards increasing values of $t$, then $S_{1}$ and $S_{2}$ should be oriented as shown so that $[2 F]=\left[S_{1}\right]+\left[S_{2}\right]$. (Note the linearity $\chi(2 F)=-4=\chi\left(S_{1}\right)+\chi\left(S_{2}\right)$ shows that $F$ is the midpoint of a face of Thurston's norm.) In Figure 6, a broken measure is assigned to each level train track $\tau_{t}$ in order to describe the broken measure on $B$. Two weights $w_{1}, w_{2}$ are sufficient to determine the broken measure on $\tau_{0}$, but for convenience we add a weight $w_{3}=w_{1}+w_{2}$. As $t$ increases, the broken measures on $\tau_{t}$ are determined by preceding ones as indicated in the sequence of frames. The final frame shows the broken measure on $\rho\left(\tau_{1}\right)$, which must be the same as the broken measure on $\tau_{0}$. Equating weights on two sectors, we get the following equations:

$$
w_{1}=\sigma_{1} \sigma_{2} w_{3}+\sigma_{1}^{2} w_{1}, \quad w_{2}=\sigma_{2}^{2} w_{3} .
$$

Eliminating $w_{3}$ using $w_{3}=w_{1}+w_{2}$ we obtain a system of equations linear in $w_{1}$ and $w_{2}$ :

$$
\begin{aligned}
\left(\sigma_{1}^{2}+\sigma_{1} \sigma_{2}-1\right) w_{1}+\left(\sigma_{1} \sigma_{2}\right) w_{2} & =0 \\
\left(\sigma_{2}^{2}\right) w_{1}+\left(\sigma_{2}^{2}-1\right) w_{2} & =0 .
\end{aligned}
$$

Since $B$ carries affine laminations this system must have a nontrivial solution for some $\sigma$. The condition for the existence of a solution is:

$$
\sigma_{1}^{2} \sigma_{2}^{2}-\sigma_{1}^{2}-\sigma_{2}^{2}-\sigma_{1} \sigma_{2}+1=0
$$

which describes $\mathcal{S}(B)$. To find the cohomology classes $x_{1} S_{1}+x_{2} S_{2}$ where the stretch factor is $\lambda$, we substitute $\lambda^{-x_{i}}$ for $\sigma_{i}$ to get

$$
\lambda^{2 x_{1}-2 x_{2}}-\lambda^{-2 x_{1}}-\lambda^{-2 x_{2}}-\lambda^{-x_{1}-x_{2}}+1=0 .
$$

Proof of Corollary 2.1. $\quad$ Let $B$ be a branched surface which fully carries the suspended lamination $L$ for fibers in the cone over the fiber face. Let us choose an arbitrary fiber $F$ whose class lies in the cone over the fiber face. Then $M=(F \times I)_{\rho}$, where $\rho$ is the monodromy. As in Example 2.2, we examine the broken measure as a 1-parameter family of broken measures on train tracks $B \cap F_{t}$ where $F_{t}=F \times t$. In $B \cap F_{0}$ we choose one weight $w_{i}$ in each sector $Z_{i}$, then all remaining weights in the sector are determined by the factors $\sigma_{i}$ associated to the surfaces $S_{i}$. Inductively, one shows that the broken measure on $\tau_{0}$ determines a broken measure on $\tau_{t}$ for all $t$. Finally one equates the weights in $\tau_{1}$ with corresponding weights $w_{i}$ in $\tau_{0}$. 


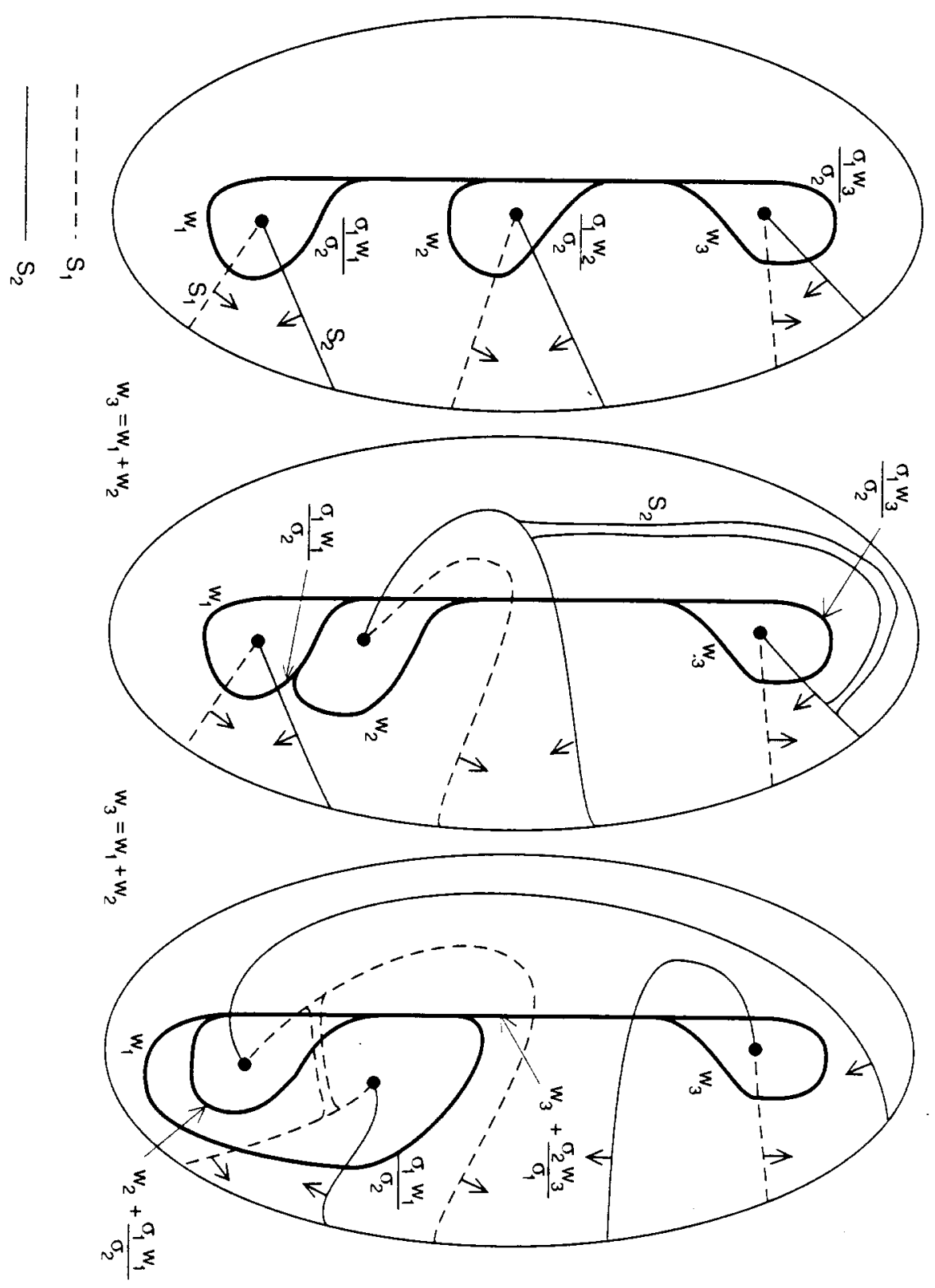

Figure 6a. 

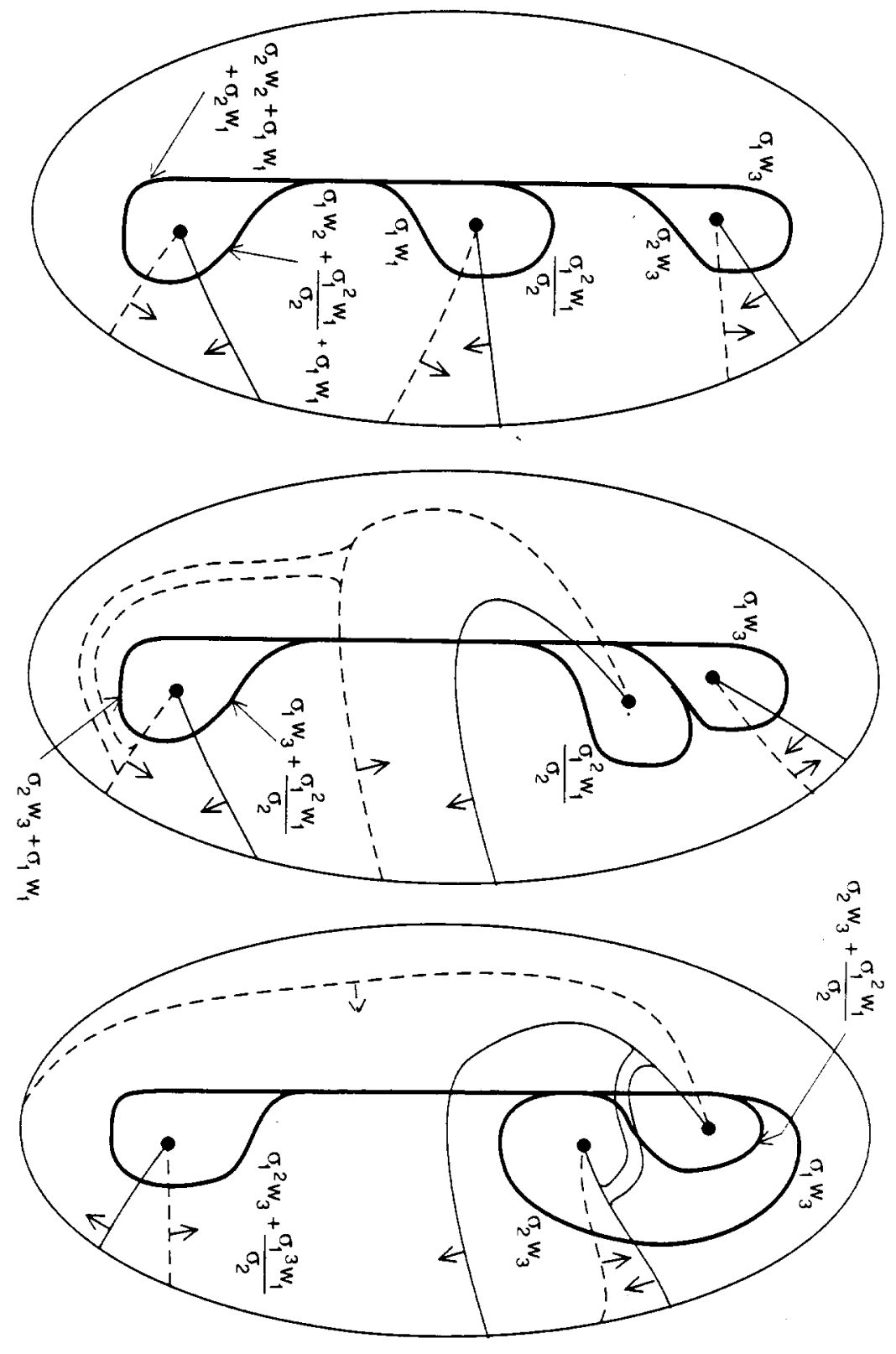

Figure $6 \mathrm{~b}$. 


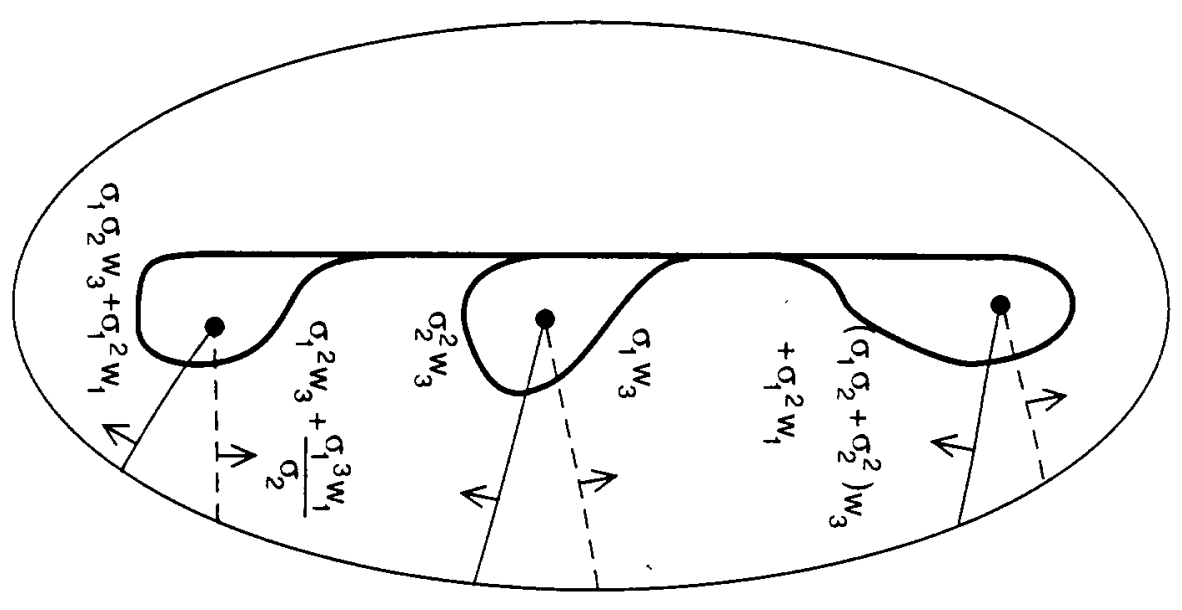

Figure 6c.

If there are $s$ sectors, then we obtain $s$ equations linear in the $s$ variables $w_{i}$, with coefficients which are polynomials in $\sigma_{i}$ 's. Solutions exist provided the determinant of the coefficient matrix is 0 . This gives a polynomial $f$ in the $\sigma_{i}$ 's which, equated to zero, defines $\mathcal{S}(B)$.

There is a simple application of the ideas in this section which is probably worth mentioning. It is motivated by [HO1]. From [HO1] we know that given a surface $F$, for every stretch homomorphism $\phi: \pi_{1}(F) \rightarrow \mathbb{R}_{+}$we have a projectivized space of affine laminations $\mathcal{P} \mathcal{A F}_{\phi}$, which is homeomorphic to the projective lamination space of the same surface, i.e. homeomorphic to a sphere of dimension $6 g-6$ when $F$ is a closed surface. Suppose that $f$ is a pseudo-Anosov homeomorphism of $F$ preserving the class $\psi=\log \phi$. Then $f$ acts on $\mathcal{P} \mathcal{A} \mathcal{F}_{\phi}$, and it is natural to ask whether there exists a pair of affine laminations invariant under $f$. If so, they will certainly be the same topologically as the stable and unstable laminations of the pseudo-Anosov homeomorphism.

Proposition 2.3. Suppose $f: F \rightarrow F$ is a pseudo-Anosov homeomorphism of a surface which fixes a class $\alpha \in H^{1}(F ; \mathbb{R})$. Then the stable lamination of $f$ has an affine structure with stretch homomorphism $e^{\epsilon \alpha} \in \operatorname{Hom}\left(\pi_{1} F ; \mathbb{R}_{+}\right)$, for some number $\epsilon$.

Proof. We consider the mapping torus $(F \times I)_{f}$, a fibered 3-manifold $M$. Now $H_{2}(M ; \mathbb{R})$ has rank at least two, with independent generators $[F]$ and 
$A$, where $A \in H_{2}(M ; \mathbb{R})$ is, roughly speaking, the mapping torus of $\alpha \in$ $H_{1}(F ; \mathbb{R})$. The class $A$ can be constructed explicitly by representing $\alpha$ by a cycle $c$, then forming a relative cycle $c \times I$ representing a class in $H_{2}(F \times$ $I, F \times \partial I ; \mathbb{R})$. This also yields a relative cycle $c \times I$ in $H_{2}\left((F \times I)_{f}, F_{0} ; \mathbb{R}\right)$ by excision, where $F_{0}$ is the surface in the mapping torus obtained by identifying $F \times 0$ with $F \times 1$ via the map $f$. But in the sequence for relative homology, we have $\partial(c \times I)=\alpha-f_{*}(\alpha)=0 \in H_{1}(F ; \mathbb{R})$, so we can form a cycle $A \in H_{2}(M ; \mathbb{R})$.

We consider the space of broken measures on a branched surface $B$ carrying the suspended stable lamination $L$ for $f$. The class $[F]$ is in the interior of the fiber face, hence we know that for rational classes which are (projectively) near $[F], L$ has affine structures corresponding to different fibrations "near" the fibration with fiber $F$. For each such affine structure, corresponding to a broken measure $(\sigma, w)$ on $B$, one has an affine structure on the stable lamination in $F$, as one easily sees by considering a broken measure on the invariant train track induced from the intersection $B \cap F_{0}$. This broken measure on the invariant train track represents an affine structure on the stable lamination $L \cap F_{0}$. If we take $[F]+\epsilon A$ as the class near $[F]$, we see that we obtain an affine structure for the stable lamination of $f$ with the required stretch homomorphism.

\section{Affine essential laminations in 3-manifolds.}

We shall see in this section that there are interesting affine laminations in 3-manifolds, other than suspended stable laminations for pseudo-Anosov homeomorphisms. Our goal here is to show that affine laminations which are also essential laminations exist commonly in 3-manifolds, even sometimes in non-Haken 3-manifolds. On the other hand, affine essential laminations are rarer than arbitrary essential laminations.

Recall that essential laminations were introduced in [GO] as a possible generalization of incompressible surfaces. The hope is that many of the theorems which hold for Haken manifolds also hold for manifolds containing essential laminations, see [GO]. These manifolds are called laminar or laminated manifolds. There are quite a number of papers which indicate that essential laminations are abundant, see $[\mathbf{D}],[\mathbf{N}],[\mathbf{R}],[\mathbf{H O 2}]$. It is not true, however, that all 3-manifolds with infinite fundamental group are laminar; the only known counterexamples are small Seifert fibered manifolds, see [B2], [Cl]. Only some of the results true for Haken manifolds are known to be true for laminar manifolds. Thus the universal cover of a laminar manifold is homeomorphic to $\mathbb{R}^{3}$, see [GO]. However, it has not been shown that any homotopy equivalence from a 3-manifold to a laminar 3-manifold 
can be replaced by a homeomorphism between the same 3-manifolds.

A manifold containing an essential measured lamination is necessarily Haken, since if the measured essential lamination is carried by an essential branched surface $B$, then the lamination can be approximated by a surface carried by the same branched surface, and the surface is then incompressible. The same is not true for affine laminations; there are non-Haken manifolds containing affine essential laminations, as we shall see. Affine essential laminations appear to be more accessible than arbitrary essential laminations; they can be described by finite data on a branched surface. The technical problems involved in proving "homotopy equivalence implies homeomorphism" may be less formidable for manifolds containing affine essential laminations

When considering affine laminations in 3-manifolds (or arbitrary manifolds) it is useful to make a distinction between two different notions of "affine." First, there is the usual notion given in our earlier definitions. We shall say a lamination $L \subset M$ is locally affine if there is a neighborhood $N$ of $L$ such that $L$ has an affine structure in $N$, i.e. that the lift of $L$ to the universal cover $\tilde{N}$ has a transverse measure $\mu$ satisfying $\alpha^{*}(\mu)=\phi(\alpha) \mu$, where $\phi$ is a stretch homomorphism and $\alpha$ is a covering translation.

Before continuing, we describe briefly the well-understood locally affine essential laminations which are constructed from suspended stable laminations for pseudo-Anosov homeomorphisms of surfaces. Given a surface $F$ with boundary, and a pseudo-Anosov homeomorphism $f$ of the surface, the mapping torus $M=(M \times I)_{f}$ has one or more torus boundary components. As we have seen, $M$ contains the suspended stable lamination, which is affine, and it is easy to check that the suspended lamination is also essential. Using, for example, [HO2] or [Wu], one can examine the effect of Dehn filling on such a lamination. The analysis shows that for most Dehn fillings the lamination remains essential. Using mapping tori of punctured tori, one can construct examples of essential locally affine laminations in closed non-Haken manifolds.

The following rather simple family of examples gives some indication that locally affine essential laminations are, in some sense, abundant.

Examples 3.1. Given any $n \times n$ irreducible Perron-Frobenius matrix $A$ with integer entries, we will construct an affine lamination with stretch factor at a primitive integer lattice class equal to the Perron-Frobenius eigenvalue $\lambda$ of the matrix. In other words, $A$ is an irreducible matrix with non-negative integer entries $a_{i j}$ and $\lambda$ is its largest eigenvalue. We construct a branched surface $B$ from a collection of planar surfaces $P_{j}, j=1, \ldots, n$. Each planar surface $P_{j}$ has $1+\sum_{i} a_{i j}$ boundary components, and we may assume that the 
planar surfaces are all oriented. One boundary component of $P_{j}$ is called the "upper" boundary circle, $\gamma_{j}$ say, and the others, $\sum_{i} a_{i j}$ in number, are called "lower" boundary circles. Now we identify $a_{i j}$ lower boundary components of $P_{j}$ with $\gamma_{i}, i=1, \ldots, n$, respecting orientation. The resulting complex is made into a branched surface $B$ with the property that wherever an upper boundary circle is attached to a lower boundary circle the union of the collars of the upper boundary circle and the lower boundary circle is smooth. The branched surface is non-generic, so it does not fit the models of Figure 2. It could be modified to fit those models, but it is preferable to leave it nongeneric.

Our next task is to embed $B$ in a 3-manifold. This is easy to do; the manifold will be constructed from products of the form $P_{j} \times I$, where $I$ is an oriented interval. Identifications are made on annuli $\partial P_{j} \times I$ according the same scheme as above, where annuli corresponding to lower boundaries are attached to annuli corresponding to upper boundaries in any order, always respecting the foliation by intervals $I$, and respecting the orientations of the intervals of the foliation. The result is a fibered neighborhood $N(B)$, which contains an embedded branched surface isomorphic to $B$. Let $N$ denote the manifold we have constructed as a fibered neighborhood. The union of annuli corresponding to upper boundaries in $\partial P_{j} \times I$ appear as a union of annuli properly embedded in $N$, and if they are all given transverse orientations opposite to those induced by the orientations of $P_{j}$ and $I$, they represent an integer lattice class in $H^{1}(N ; \mathbb{R})$. We let $S$ denote the union of the oriented annuli, and we let $[S] \in H^{1}(N ; \mathbb{R})$ represent the class. In terms of the homotopy equivalent branched surface, the class is represented by the union of the branch curves transversely oriented toward the single-sheeted side of the branch curves. We could represent the class by the same curves pushed off the branch locus to the single sheeted side, but it is preferable to leave them as they are. Let the $n$-vector $w$, with entries $w_{i}$, be the eigenvector of $A$ corresponding to the Perron-Frobenius eigenvalue $\lambda$ of $A$. We easily construct a broken measure with weight $w_{i}$ on $P_{i}$ and with one factor $\sigma=\lambda$ associated to the transversely oriented surface $S$. Then the stretch factor of the affine lamination $L$ determined by this broken measure at the class $[S]$ is $\lambda$.

Finally, it remains to re-embed the affine lamination constructed above in a closed manifold as an affine essential lamination. According to Proposition 3.1 of [HO2], if 2-handles are attached to $N=N(B)$ in a sufficiently complex way to yield a manifold with sphere boundary components, and boundary spheres are capped off with 3 -handles to obtain a manifold $M$, then the branched surface $B$ is essential in $M$ and hence $L$ is also essential in $M$. For any manifold $M$ constructed in this way, we have a locally affine essential 
lamination $L$ in $M$.

We will not do the analysis of all affine laminations carried by $B$, but the special case done in Example 1.9 gives some indication of what to expect.

The irreducibility of the matrix $A$ can be interpreted as well. It implies that the lamination $L$ is minimal.

Notice that the general procedure just described can be applied to (almost) any closed affine lamination embedded in any 3-manifold to re-embed it as a locally affine essential lamination in a closed manifold. Thus one can construct many families of locally affine essential laminations. Let us record the results of the previous example as a proposition:

Proposition 3.2. Let $\lambda$ be the largest eigenvalue of an irreducible PerronFrobenius matrix with integer entries. Then there exists a 3-manifold $N$, a minimal, affine, essential lamination $L$ in $N$, and a primitive integer lattice class $[S] \in H^{1}(N ; \mathbb{R})$ such that the stretch factor of $L$ at $[S]$ is $\lambda$. Further, $N$ can be embedded in a closed manifold $M$, such that $L$ is locally affine and essential in $M$.

Question: Which algebraic numbers can be realized as stretch factors of affine laminations in 3-manifolds?

We come now to affine laminations representing homology. Just as a class in $H_{2}(M ; \mathbb{Z})$ for a closed manifold $M$ can be represented by a surface, so classes in real homology with twisted coefficients, $H_{2}^{\theta}(M ; \mathbb{R})$, can be represented by affine laminations, where $\theta: \pi_{1}(M) \rightarrow \mathbb{R}_{*}$ is a homomorphism to the non-zero reals with multiplication, and describes the twisting of coefficients.

We give a definition of $H_{2}^{\theta}(M ; \mathbb{R})$. It is defined in terms of certain infinite chains in the cover $\hat{M}$ of $M$ corresponding to $\operatorname{ker}(\theta)$. Let $C_{*}^{\theta}(M)$ denote infinite chains $c$ in $\hat{M}$ satisfying, for every $\alpha \in \pi_{1}(M), \alpha_{*}(c)=\theta(\alpha) \cdot c$. With the usual boundary operator, this defines a homology, which we shall call affine homology with twisting homomorphism $\theta$, denoted $H_{*}^{\theta}(M ; \mathbb{R})$. Although the chains in the chain complex are infinite, if we use singular chains, we may assume that each chain involves only the translates of finitely many singular simplices in $M$.

If $M$ is a knot exterior $M=S^{3}-\stackrel{\circ}{N}(K)$, then there are few possibilities for $\theta$. In fact, $\log (|\theta|) \in \operatorname{Hom}\left(\pi_{1}(M), \mathbb{R}_{+}\right)$can be interpreted as a class in $H^{1}(M ; \mathbb{R})$, which is generated by the Seifert surface, hence $\theta= \pm e^{\psi}$ where $\psi$ is a class in $H^{1}(M ; \mathbb{R})$. The class $\psi$ must be $x[F]$, where $x \neq 0$ is a real number and $F$ is a Seifert surface. The homomorphism $\theta$ is defined by $\theta(\alpha)=\sigma^{\langle\alpha, F\rangle}$, where $\sigma= \pm e^{x}$. In this setting, we also denote $H_{2}^{\theta}(M ; \mathbb{R})$ by $H_{2}^{\sigma}(M ; \mathbb{R})$. 
Proposition 3.3. Let $M$ be a knot exterior $M=S^{3}-\stackrel{\circ}{N}(K)$. Then $H_{2}^{\sigma}(M ; \mathbb{R})$ is non-trivial if and only if $\sigma \neq 0$ is a real root of the Alexander polynomial of $K$.

Note. This is a well-known result. Allen Hatcher showed me the following proof.

Proof. Let $\hat{M}$ be the $\mathbb{Z}$-covering space of $M$, with $t$ a generating deck transformation. Then there is a short exact sequence of chain complexes (with real coefficients throughout) as follows:

$$
0 \longrightarrow C_{*}(\hat{M}) \stackrel{\sigma t-1}{\longrightarrow} C_{*}(\hat{M}) \stackrel{\Sigma}{\longrightarrow} C_{*}^{\sigma}(M) \longrightarrow 0 .
$$

Here $\Sigma(c)=\sum_{n \in \mathbb{Z}} \sigma^{n} t^{n} c$, and $C_{*}^{\sigma}(M)$ is the chain complex described above, whose homology is $H_{2}^{\sigma}(M ; \mathbb{R})$. The short exact sequence gives a long exact sequence whose relevant part is:

$$
0=H_{2}(\hat{M}) \longrightarrow H_{2}^{\sigma}(M) \longrightarrow H_{1}(\hat{M}) \stackrel{\sigma t-1}{\longrightarrow} H_{1}(\hat{M}) .
$$

So if $H_{2}^{\sigma}(M)$ is non-trivial, it is the eigenspace for the eigenvalue $\sigma^{-1}$ of the transformation $t: H_{1}(\hat{M}) \rightarrow H_{1}(\hat{M})$. The characteristic polynomial is the Alexander polynomial $\Delta(t)$, see $[\mathbf{R l}]$.

For any 3 -manifold $M$, it is not difficult to represent a class in $H_{2}^{\theta}(M ; \mathbb{R})$ by an affine lamination. If we use simplicial homology, such a class is defined by an infinite chain in $\hat{M}$, as in the definition, with the coefficients for 2 simplices all determined by the coefficients on the preferred lifts of simplices lying, say, in one fundamental domain. If $s$ is an oriented 2-simplex in $\hat{M}$ with coefficient $c$, we construct an embedded product family of thickness $|c|$ in a neighborhood of the simplex. According to the sign of $c$, the leaves of this measured product foliation obtain an orientation. Now we can join these product families along neighborhoods of 1-simplices and 0 -simplices, in a canonical way respecting orientation and measure, to obtain an embedded equivariant measured lamination in $\hat{M}$ representing the class. Projecting to $M$, we obtain an affine lamination representing the class.

Although it is elementary to represent a class in $H_{2}^{\theta}(M ; \mathbb{R})$ by an affine lamination, it is much more difficult, at least in theory, to represent such a class by an affine essential lamination. In the following example we shall use Proposition 3.3 to find a nontrivial class in affine homology, and describe an affine essential lamination representing the class.

Example 3.4. Examination of knot tables, [Rl], shows that the Stevedore knot, $6_{1}$ on the tables, has an Alexander polynomial with real roots 

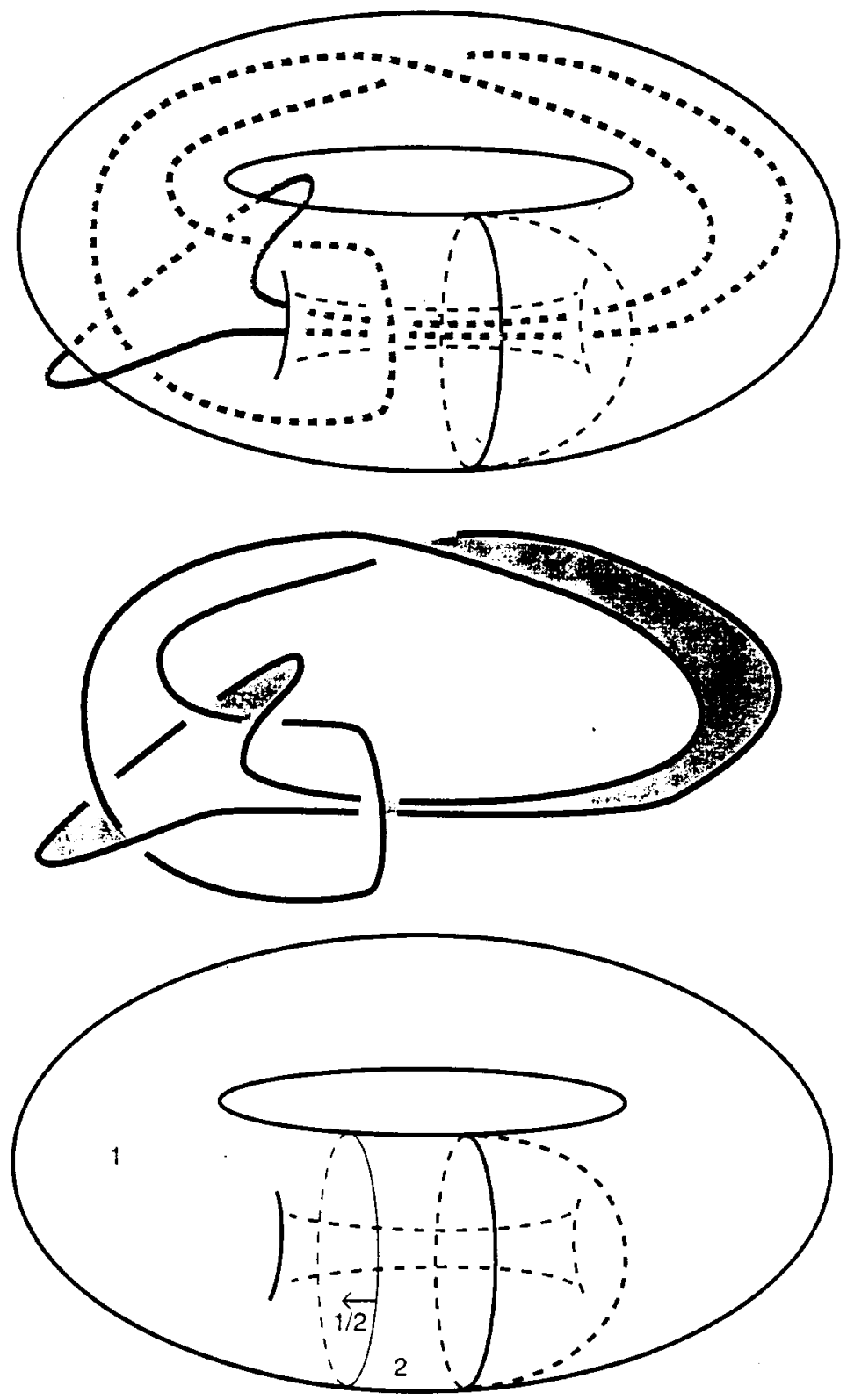

Figure 7. 
$\sigma=1 / 2,2$. Accordingly, there must be a nontrivial class in $H_{2}^{1 / 2}(M)$, where $M$ is the knot exterior. Using something like a normal surface theory adapted to knot exteriors, it is possible to construct a transversely oriented affine lamination representing this class, as shown in Figure 7 . The affine lamination is shown as a broken measure on a branched surface $B$ embedded in the knot exterior. A Seifert surface is shown, which intersects $B$ in the curve where the measure has a discontinuity. The branched surface and lamination is exactly the same as that of Example 1.9. The embedding in $S^{3}$ is constructed by first embedding a torus in the standard way; then attaching a meridian disc; then cutting two holes, one in the meridian disc and one in the torus and joining the boundaries with a tube. It is easy to verify that the branched surface is essential, which implies that the lamination is essential. Furthermore, the branched surface has the property that $\partial_{h} N(B)$ contains two distinct (non-isotopic in $\partial_{h} N(B)$ ) curves which are isotopic in $M$ to a meridian curve in $\partial M$. Applying well-known results, e.g. [HO2] or [Wu], about the effect of Dehn filling on essential laminations, one concludes that for every non-trivial surgery the lamination remains essential in the filling manifold. In the filling manifold, the lamination is locally affine and essential. The Stevedore knot is a 2-bridge knot, and Dehn surgery on 2-bridge knots yields non-Haken manifolds for all but finitely many Dehn surgeries, so this gives more examples of non-Haken manifolds which contain affine essential laminations.

For purposes of calculation, a different projection of the knot was used, namely the projection shown in the knot tables. In that projection, the branched surface carrying the affine lamination appears more complex, but also perhaps more interesting. Mark Brittenham used the branched surface in the original projection to construct new examples of essential laminations, see [B1]. Figure 7 shows the simplest possible view of the embedding of the branched surface, with an adapted view of the knot.

It is possible, using the same methods, to construct laminations representing any affine homology class in a knot exterior. A class in $H_{2}^{\sigma}(M ; \mathbb{R})$ yields an affine lamination with stretch homomorphism given by $\phi(\alpha)=|\sigma|^{\langle\alpha, F\rangle}$, where $F$ is the Seifert surface. Though it is probably easy, in every example of such a class in a knot exterior, to construct an affine essential lamination representing the class, the following question remains open.

Question: Given any manifold $M$ and a class in $H_{2}^{\theta}(M ; \mathbb{R})$, can the class be realized as an affine essential lamination? 


\section{References}

[B1] M. Brittenham, Persistently laminar tangles, preprint, 1995.

[B2] _ Essential laminations in Seifert fibered spaces, Topology, 32 (1993), 61-85.

[Ca] A. Candel, Laminations with transverse structure, preprint.

[C1] J. Christy, Intransitive Anosov flows on three-manifolds, thesis, University of California, Berkeley, 1984.

[C2] , Branched surfaces and attractors I: dynamic branched surfaces, Trans. Am. Math. Soc., 336(2) (1993), 759-784.

[Cl] W. Claus, Essential laminations in closed Seifert-fibered spaces, thesis, University of Texas, Austin, 1991.

[D] C. Delman, Essential laminations and Dehn surgery on 2-bridge knots, Topology and its Applications, 63 (1995), 201-221.

[FLP] A. Fathi, F. Laudenbach and V. Poenaru, Travaux de Thurston sur les surfaces, Asterisque, 66-67.

[F1] D. Fried, Flow equivalence, hyperbolic systems and a new zeta function for flows, Comment. Math. Helv., 57 (1982), 237-259.

[F2] _ Growth rate of surface homeomorphisms and flow equivalence., Ergodic theory and dynamical systems, 5 (1985), 539-563.

[GO] D. Gabai and U. Oertel, Essential laminations in 3-manifolds, Annals of Math., 130 (1989), 41-73.

[HO1] A. Hatcher and U. Oertel, Affine lamination spaces for surfaces, Pacific J. of Math., 154(1) (1989), 87-101.

[HO2] - Full laminations in 3-manifolds, Math. Proc. Cambr. Phil. Soc., 119 (1996), 73-82.

[L] I. Liousse,Feuilletages transversalement affines des surfaces et actions affines de groupes sur les arbres réels, Thèse, Université Paul Sabatier, 1994.

[M] L. Mosher, Dynamical systems and the homology norm of a 3-manifold, II, Invent. Math., 107 (1992), 243-281.

[N] R. Naimi, Essential laminations in 3-manifolds obtained by surgery on 2-bridge knots, Proceedings (Haifa, 1992), Contemp. Math., 164, Collection: Geometric Topology, 183-186.

[O1] U. Oertel, Homology branched surfaces: Thurston's norm on $\mathrm{H}_{2}(M)$, LMS Lecture Note Series, 112, Low Dimensional Topology and Kleinian Groups, 1986.

[O2] Incompressible branched surfaces, Inventiones Mathematicae, 76 (1984), $35-41$.

[O3] Measured laminations in 3-manifolds, Transactions AMS, 305(2) (1988), 531-573.

[OP] U. Oertel and A. Papadopoulos, Affine foliations and covering hyperbolic structures, preprint.

[Rb] R. Roberts, Constructing taut foliations, thesis, Cornell Univ., 1992.

[Rl] D. Rolfsen, Knots and links, Publish or Perish, 1976.

[T] W.P. Thurston, A norm for the homology of 3-manifolds, Memoirs of the AMS, 339, (1986). 
[Wi] R. Williams, Expanding attractors, Pub. Math. I.H.E.S., 43 (1974), 169-203.

[Wu] Ying-Qing Wu, Essential laminations in surgered 3-manifolds, Proc. A.M.S., 115 (1992), 245-249.

Received July 17, 1996.

RUTGERS UNIVERSITY

NEWARK, NJ 07102

E-mail address: oertel@andromeda.rutgers.edu 